\title{
Hysteresis-based Switching Observers for Linear Systems Using Quadratic Boundedness
}

\author{
Angelo Alessandri ${ }^{a}$ Ricardo G. Sanfelice ${ }^{b}$ \\ ${ }^{\text {a } U n i v e r s i t y ~ o f ~ G e n o a ~(D I M E), ~ V i a ~ O p e r a ~ P i a ~ 15,16145 ~ G e n o a, ~ I t a l y ~}$ \\ ${ }^{\mathrm{b}}$ Department of Electrical and Computer Engineering, University of California, Santa Cruz, CA 95064, USA
}

\begin{abstract}
Switched-gain observers are investigated for the purpose of estimating the state of linear systems affected by bounded noises. Under mild assumptions, hybrid observers with gain switching are proposed and provided with stability analysis based on quadratic boundedness for the estimation error. Such observers are designed by solving optimization problems aimed at minimizing upper bounds on the estimation error in such a way as to get the smallest invariant set. The effectiveness of the proposed approach is evaluated with some numerical case studies.
\end{abstract}

Key words: hybrid observers, hysteresis, quadratic boundedness.

\section{Introduction}

The control of switching systems has been studied in the literature in great detail from the theoretical point of view and extensively used in many application areas. By contrast, estimation of dynamic systems has not received the same amount of attention in the presence of switching that may regard the state equation and/or observer structure. In this paper, we investigate the problem of constructing hysteresis-based switched-gain state observers for linear systems. We will show how to reach this goal by formulating the problem in the hybrid systems framework and treating the proposed estimators as hybrid observers with specific additional variables to orchestrate the gain switching.

The first pioneering ideas on the use of switching for estimating the state of dynamic systems are reported in $[23,25]$. In [23], the motivation for adopting the gain switching is to increase robustness. By contrast, the contribution of [25] lies in pointing out the advantage that stems from the use of a high gain in the transient in such a way as to ensure a large bandwidth, while keeping a low gain at steady state to reduce the effect of measurement noises with a smaller bandwidth. Switched-gain

\footnotetext{
^ This paper was not presented at any IFAC meeting. Corresponding author A. Alessandri. Tel. +39-010-3356007.

Email addresses: angelo.alessandri@unige.it (Angelo Alessandri), ricardo@ucsc. edu (Ricardo G. Sanfelice).
}

observers allow to increase the rate of convergence with finite-time stability in a noise-free setting, while guaranteeing input-to-state stability of the estimation error with respect to system and measurement noises [17], and in [27] they are shown to overcome the difficulties to deal with estimation for non-monotonic nonlinear systems. Hybrid observers based on switching between local and global estimators are proposed in [7].

Hybrid estimation arises naturally when dealing with hybrid systems, i.e., with dynamics that exhibit characteristics of both continuous-time and discrete-time systems, suitably combined to model complex plants in which such different dynamic behaviors interact [20,32]. However, the "hybridization" of estimators may provide helpful features as well. Hybrid estimators are presented in $[22,28]$ with finite-time stability properties. In [18], state estimation is performed with measurements available at a variable frequency. Hybrid observers based on reset to reduce peaking are proposed in $[5,26]$. Conditions ensuring the stability of the estimation error provided by observers for general hybrid linear systems are presented in [10]. A hybrid scheme is presented in [9] to design observers with different sets of coordinates with respect to the system to estimate. Hybrid observers with periodic jumps are considered in $[29,30]$. In [6], the construction of observers with state trajectories permanently remaining in compact convex sets is addressed within a hybrid framework, while ensuring robustness to disturbances. 
In this paper, we deal with switched-gain observers for linear systems by providing novel results that complete those of $[2,3]$. More specifically, in [2] Luenberger observers are proposed to estimate the state variables of various kinds of dynamic systems subject to bounded noises by relying on the notion of quadratic boundedness (QB) [15], which allows to account for the knowledge on the noise bounds and can be treated by using linear matrix inequalities (LMIs) [14]. Using QB, we design observers that may switch between two different gains depending on the residuals given by the output error. The gain switching may be subject to a preset hold time to reduce wigwag. To the authors' knowledge, this is the first work that addresses the problems of both studying the invariance properties of the estimation error and investigating the design through an effective choice of the gains for such observers, as compared with the few approaches already available in the literature (see, e.g., [17]). The presence of discontinuity in the observer structure may be beneficial in terms of robustness and even insensitivity to disturbances $[8,19]$.

Set invariance is the theoretical framework in which we may formulate and solve the problem to construct switched-gain observers with the goal of getting the smallest invariant set for the estimation error $[11,12]$. In this context, QB allows to construct ellipsoidal invariant sets and find upper bounds on the estimation error $[2,15]$. In addition, the knowledge of the bounds on the system and measurement disturbances is incorporated into the observer design, which can be accomplished by using bilinear matrix inequalities (BMIs). The BMI design conditions are solved by means of an LMI-based approach with grid search optimization [13].

The paper is organized as follows. In Section 2 we will present the proposed approach based on $\mathrm{QB}$ and how to design constant-gain observers for linear time-invariant systems. This allows to motivate the extension of the approach to construct switched-gain observers, as described in Sections 3. Numerical results are shown in Section 4. The conclusions are drawn in Section 5.

Given the column vectors $x$ and $y$, let us define $(x, y):=\left[x^{\top}, y^{\top}\right]^{\top}, \mathbb{R}_{>0}:=(0,+\infty), \mathbb{R}_{\geq 0}:=[0,+\infty)$, and $\mathbb{N}_{\geq 0}:=\{0,1,2, \ldots\}$. The minimum and maximum eigenvalues of a symmetric matrix $P \in \mathbb{R}^{n \times n}$ are denoted by $\lambda_{\min }(P)$ and $\lambda_{\max }(P)$, respectively; in addition, $P>0(P<0)$ means that it is also positive (negative) definite. For a generic matrix $M \in \mathbb{R}^{n \times m}$, let us define $|M|:=\left(\lambda_{\max }\left(M^{\top} M\right)\right)^{1 / 2}=\left(\lambda_{\max }\left(M M^{\top}\right)\right)^{1 / 2}$ and thus $|v|:=\left(v^{\top} v\right)^{1 / 2}$ denotes the Euclidean norm of a vector $v \in \mathbb{R}^{n}$. The diagonal matrix with $v_{1}, \ldots, v_{n}$ on the diagonal is denoted by $\operatorname{diag}(v) \in \mathbb{R}^{n \times n}$. The symbol $1^{\top}$ denotes a row vector of appropriate dimension with every element equal to 1 .

We refer to the hybrid system modeling framework pro- posed in [20]. More specifically, consider

$$
\begin{aligned}
& \dot{x}=f(x, d), \quad(x, d) \in \mathcal{C} \\
& x^{+}=g(x, d), \quad(x, d) \in \mathcal{D}
\end{aligned}
$$

where $d$ is an external input and $x$ is the state of the system; $\mathcal{C}$ and $\mathcal{D}$ are closed and called flow set and jump set, respectively. The state changes according to the differential equation (1a) if it belongs to the flow set, while $x$ is subject to instantaneous change if it is in the jump set with $x^{+}$denoting the value of the state after this change according to $(1 \mathrm{~b})$.

We call hybrid time domain any subset $E$ of $\mathbb{R}_{\geq 0} \times \mathbb{N}_{\geq 0}$ such that, for each $(T, J) \in E, E \cap([0, T] \times\{0,1, \ldots, J \bar{J}\})$ can be written in the form $\cup_{j=0}^{J}\left(\left[t_{j}, t_{j+1}\right], j\right)$ for some some finite sequence of times $0=t_{0} \leq t_{1} \leq t_{2} \leq \ldots \leq$ $t_{J}$. Let a hybrid $\operatorname{arc} \phi(t, j)$ be a solution to the hybrid system (1a)-(1b) with the initial condition $\phi(0,0) \in \overline{\mathcal{C}} \cup \mathcal{D}$ and hybrid input $d(t, j)$ if $t \mapsto \phi(t, j)$ is locally absolutely continuous for each $j, t \mapsto d(t, j)$ is Lebesgue measurable and locally essentially bounded for each $j$, $\operatorname{dom} \phi=\operatorname{dom} d$, and

- for all $j \in \mathbb{N}_{\geq 0}$ and almost all $t$ such that $(t, j) \in$ $\operatorname{dom} \phi,(\phi(t, j), d(t, j)) \in \mathcal{C}$ and $\dot{\phi}(t, j)=f(\phi(t, j)$, $d(t, j))$;

- for all $(t, j) \in \operatorname{dom} \phi$ such that $(t, j+1) \in \operatorname{dom} \phi$, $(\phi(t, j), d(t, j)) \in \mathcal{D}$ and $\phi(t, j+1)=g(\phi(t, j), d(t, j))$.

Given a hybrid input, the hybrid arc solving (1) is said maximal if it cannot be extended, i.e., it is not a truncated version of another solution; it is called complete if its domain is unbounded.

\section{Observers Based on Quadratic Boundedness}

Let us consider plants described by

$$
\begin{aligned}
& \dot{x}=A x+B u+D w \\
& y=C x+E w
\end{aligned}
$$

where $x \in \mathbb{R}^{n}$ is the state, $u \in \mathbb{R}^{p}$ is the control input, $y \in \mathbb{R}^{m}$ is the output; $w \in \mathbb{R}^{s}$ is the vector of system and measurement disturbances; $A \in \mathbb{R}^{n \times n}, B \in \mathbb{R}^{n \times p}$, $C \in \mathbb{R}^{m \times n}, D \in \mathbb{R}^{n \times s}$, and $E \in \mathbb{R}^{m \times s}$. Moreover, we assume that each disturbance $t \mapsto w_{i}(t) \in \mathbb{R}$ is Lebesgue measurable, essentially bounded, and such that $\left|w_{i}(t)\right| \leq$ $1, i=1, \ldots, s$ for almost all $t \geq 0$. This assumption on the upper bound of the noises does not entail loss of generality since the related entries in $D$ and/or $E$ can be scaled in case of different bounds.

The Luenberger observer for (2) providing an estimate $\hat{x} \in \mathbb{R}^{n}$ of $x$ is given by

$$
\dot{\hat{x}}=A \hat{x}+B u+L(y-C \hat{x})
$$


where $L \in \mathbb{R}^{n \times m}$ is the observer gain. If the pair $(A, C)$ is detectable, an observer with an exponentially stable estimation error $e:=x-\hat{x} \in \mathbb{R}^{n}$ in a noise-free setup can be constructed by choosing $L$ such that $A-L C$ is Hurwitz. In the presence of noise, from (2) and (3) we obtain

$$
\dot{e}=(A-L C) e+(D-L E) w
$$

and, following the approach in [2], the stability of the estimation error can be studied by using QB. Generally speaking, (4) is said to be invariant with Lyapunov function $V: \mathbb{R}^{n} \rightarrow[0,+\infty)$ if

$$
V(e)>1 \Rightarrow \dot{V}(e, w)<0, \forall w \in[-1,1]^{s} .
$$

Moreover, the set $\mathcal{E}_{V}:=\left\{e \in \mathbb{R}^{n}: V(e) \leq 1\right\}$ is positively invariant, contains the reachable set from the origin, and is attractive (i.e., if the error is outside of $\mathcal{E}_{V}$, it approaches $\mathcal{E}_{V}$ in finite time) if (5) is satisfied.

If (5) holds for a quadratic Lyapunov function $V(e)=$ $e^{\top} P e$ with $P>0$ [15], (4) is said to be quadratically bounded; moreover, (5) becomes

$$
\begin{aligned}
e^{\top} P e>1 & \Rightarrow 2 e^{\top} P((A-L C) e \\
& +(D-L E) w)<0, \forall w \in[-1,1]^{s}
\end{aligned}
$$

In such a case the invariant, attractive set is given by $\mathcal{E}_{P}:=\left\{e \in \mathbb{R}^{n}: e^{\top} P e \leq 1\right\}$. In addition, any error signal $t \mapsto e(t)$ can be bounded from above as follows:

$$
|e(t)|^{2} \leq \frac{1}{\lambda_{\min }(P)} \max \left\{e(0)^{\top} P e(0), 1\right\}
$$

for all $t \geq 0$ and

$$
\limsup _{t \rightarrow+\infty}|e(t)| \leq \frac{1}{\sqrt{\lambda_{\min }(P)}}
$$

The following result provides a sufficient condition to ensure the QB of the estimation error (see [2]).

Theorem 1 If there exist a symmetric matrix $P>0$, $Y \in \mathbb{R}^{n \times m}, \alpha \in \mathbb{R}^{s}$ with $\alpha_{i}>0, i=1, \ldots, s$, and $a$ scalar $\beta>0$ such that

$$
\begin{aligned}
& \left(\begin{array}{cc}
A^{\top} P-C^{\top} Y^{\top}+P A-Y C+\beta P & P D-Y E \\
\star & -\operatorname{diag}(\alpha)
\end{array}\right)<0 \\
& \sum_{i=1}^{s} \alpha_{i}-\beta \leq 0 .
\end{aligned}
$$

the estimation error given by the observer (3) with gain $L=P^{-1} Y$ is quadratically bounded.
The parameters $\alpha_{i}$ and $\beta$ are needed to account for noise bounds as required by the $\mathrm{QB}$ invariance conditions. In practice, such parameters as well as the other ones introduced later are Lagrange multipliers that allow to account for constraints or other conditions (see, for details, the S-procedure [14, p. 23] and the references therein). Since $-\beta$ is also the exponential coefficient governing the decrease of the estimation error in the noise-free setting, one can proceed with the design first by finding the maximum of $\beta>0$ and then maximizing the minimum eigenvalue of $P$ at the price of some reduction of $\beta[2-4]$.

Remark 1 Concerning the feasibility of the conditions stated in Theorem 1, let us show that such conditions can be easily satisfied without additional assumptions except the detectability of the pair $(A, C)$. Toward this end, let us replace $P=p_{0} \bar{P}$ with the matrix $\bar{P}>0$ and scalar $p_{0}>0$ in (9), thus obtaining

$$
\left(\begin{array}{cc}
p_{0}\left(A_{L}^{\top} \bar{P}+\bar{P} A_{L}+\beta \bar{P}\right) & p_{0}(\bar{P} D-\bar{P} L E) \\
\star & -\operatorname{diag}(\alpha)
\end{array}\right)<0
$$

where $A_{L}:=A-L C$ for the sake of brevity. If we choose $\bar{L} \in \mathbb{R}^{m}$ such that $A_{\bar{L}}$ is Hurwitz, there exist $\bar{P}>0$ such that $A_{\bar{L}}^{\top} \bar{P}+\bar{P} A_{\bar{L}}<0$ and, for the sign-preserving property of continuous functions, $\bar{\beta}>0$ sufficiently small such that $A_{\bar{L}}^{\top} \bar{P}+\bar{P} A_{\bar{L}}+\bar{\beta} \bar{P}<0$. Moreover, let us choose $\bar{\alpha}_{i}=\bar{\beta} / s, i=1, \ldots, s$, in such a way as to satisfy (10). Therefore, using the Schur lemma, it follows that (11) is equivalent to

$$
\begin{aligned}
& p_{0}\left(A_{\bar{L}}^{\top} \bar{P}+\bar{P} A_{\bar{L}}+\bar{\beta} \bar{P}\right) \\
& +p_{0}^{2}(\bar{P} D-\bar{P} \bar{L} E)^{\top}(\bar{P} D-\bar{P} L E) \frac{\bar{\beta}}{s}<0,
\end{aligned}
$$

which holds by choosing a sufficiently small $p_{0}>0$, thus proving the feasibility of (9) and_(10) with $P=p_{0} \bar{P}$, $Y=\left(p_{0} \bar{P}\right)^{-1} \bar{L}, \beta=\bar{\beta}$, and $\alpha_{i}=\bar{\beta} / s, i=1, \ldots, s$.

Clearly, it is preferable to impose a small steady-state error by increasing $\lambda_{\min }(P)$ as much as possible, i.e., with the smallest invariant set for the estimation error. Toward this end, in [2] an LMI-based design procedure is proposed aimed at maximizing $\lambda$ subject to the constraint $P>\lambda I$, i.e.,

$$
\max _{P>0 ; Y ; \alpha \in \mathbb{R}_{>0}^{s} ; \beta, \lambda>0} \lambda \text { s.t. } P>\lambda I,(9),(10) .
$$

The solution of this problem provides a gain $L$ and the steady-state bound $\rho_{e}:=1 / \sqrt{\lambda_{\min }(P)}$ according to (8), which corresponds to the minimization of the regime value of the estimation error. The value of $\rho_{e}$ certifies 
the effectiveness of the design and can be used for the purpose of decision. We can evaluate an upper bound on the norm of the residual $r:=y-C \hat{x}$ by noting that

$$
\begin{aligned}
|r| & =|y-C \hat{x}|=|C e+E w| \leq|C e|+|\bar{E} \bar{w}| \\
& \leq|C| \rho_{e}+\sqrt{k}|\bar{E}|
\end{aligned}
$$

where $\bar{E} \in \mathbb{R}^{m \times k}$ is the matrix made by the non-null columns of $E$ and $\bar{w} \in[-1,1]^{k}$ is the vector of the corresponding components of $w$, with the integer $k \leq s$. Thus, it follows that $|r|$ has to be lower than

$$
\theta_{\mathrm{th}}:=|C| \rho_{e}+\sqrt{k}|\bar{E}|>0
$$

at steady state. Since the design procedure based on (12) aims at minimizing the asymptotic upper bound while neglecting the transient behavior, a switched-gain observer turns out to be well-suited to trading between these two different goals. The switching rule can be set on the basis of (13), in that it is driven by evaluating the norm of the residual in real time. Thus, in the next section two different switched-gain estimators will be analyzed.

\section{Switched-gain Observers}

A hybrid switched-gain observer is considered with a discrete state $q$ that remains constant during flows and takes values 1 or 2 according to a hysteresis mechanism based on the norm of the residual ) $=y-C \hat{x}$. More specifically, $q=1$ is associated with "small" residuals, while $q=2$ corresponds to "high" residuals.

In line with recently developed approaches to estimation based on hybrid modeling [5-7,9,18,22, 27-30], the proposed observer is described by

$$
\left.\begin{array}{ll}
\begin{array}{l}
\dot{\hat{x}}=A \hat{x}+B u+L_{q}(y-C \hat{x}) \\
\dot{q}=0
\end{array} & \{(\hat{x}, q, y, u, w) \in \mathcal{C} \\
\hat{x}^{+}=\hat{x} & \\
q^{+}=3-q
\end{array}\right\}(\hat{x}, q, y, u, w) \in \mathcal{D}
$$

where $y=C e+E w, \mathcal{C}=\mathcal{C}_{1} \cup \mathcal{C}_{2}$, and $\mathcal{D}=\mathcal{D}_{1} \cup \mathcal{D}_{2}$ with

$$
\begin{aligned}
\mathcal{C}_{1}:=\left\{(\hat{x}, q, y, u, w) \in \mathbb{R}^{n} \times\{1,2\} \times \mathbb{R}^{m} \times \mathbb{R}^{p}\right. \\
\left.\times[-1,1]^{s}:|y-C \hat{x}| \geq \delta_{1} \theta_{\mathrm{th}}, q=1\right\}, \\
\mathcal{D}_{1}:=\left\{(\hat{x}, q, y, u, w) \in \mathbb{R}^{n} \times\{1,2\} \times \mathbb{R}^{m}\right. \\
\left.\quad \times \mathbb{R}^{p} \times[-1,1]^{s}:|y-C \hat{x}| \leq \delta_{1} \theta_{\mathrm{th}}, q=1\right\}, \\
\mathcal{C}_{2}:=\left\{(\hat{x}, q, y, u, w) \in \mathbb{R}^{n} \times\{1,2\} \times \mathbb{R}^{m}\right. \\
\left.\quad \times \mathbb{R}^{p} \times[-1,1]^{s}:|y-C \hat{x}| \leq \delta_{2} \theta_{\mathrm{th}}, q=2\right\} \\
\mathcal{D}_{2}:=\left\{(\hat{x}, q, y, u, w) \in \mathbb{R}^{n} \times\{1,2\} \times \mathbb{R}^{m}\right.
\end{aligned}
$$

$$
\left.\times \mathbb{R}^{p} \times[-1,1]^{s}:|y-C \hat{x}| \geq \delta_{2} \theta_{\mathrm{th}}, q=2\right\},
$$

and $\delta_{1}, \delta_{2} \in(0,1), \delta_{1}<\delta_{2}$ (the parameter $\theta_{\text {th }}$ is defined in (13)). Fig. 1 pictorially shows the switching mechanism.

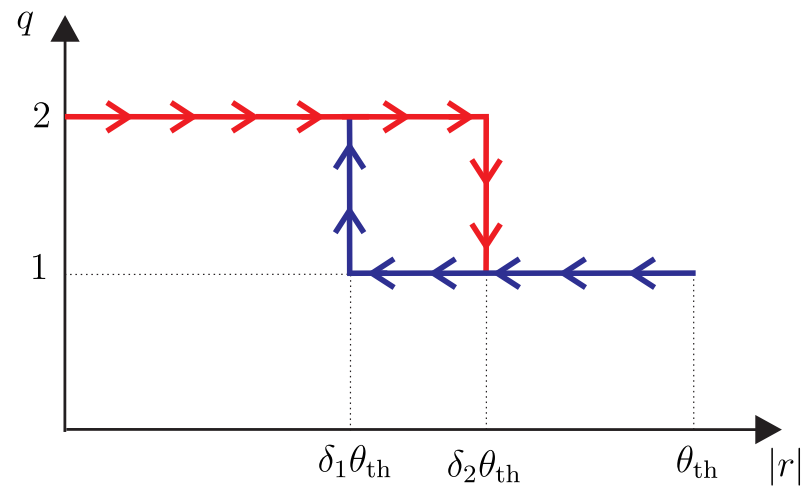

Fig. 1. Switching rule based on hysteresis.

The interconnection between the plant in (2) and the hybrid observer in (14) results in the hybrid system

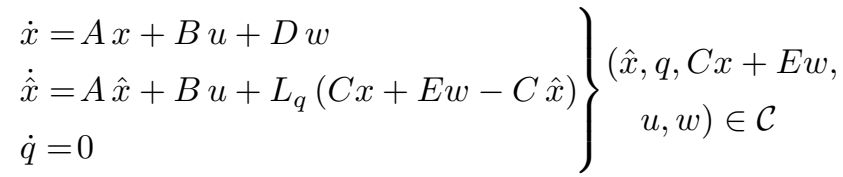

$$
\begin{aligned}
& x^{+}=x \\
& \hat{x}^{+}=\hat{x} \\
& q^{+}=3-q \\
& \left\{\begin{array}{l}
(\hat{x}, q, C x+E w \\
u, w) \in \mathcal{D}
\end{array}\right.
\end{aligned}
$$

with state $(x, \hat{x}, q)$, control input $u$, and disturbance input $w$. When the input $d=(u, w)$ is zero, this hybrid system is well-posed, in the sense of [20, Definition 6.29, p. 133]. In particular, well-posed nominal hybrid systems with asymptotically stable compact sets are such that asymptotic stability is robust to small perturbations; see $[20$, Theorem 7.21 , p. 152]. The following proposition establishes well-posedness of (15) as well as existence of solutions.

Proposition 1 For zero inputs, the hybrid system resulting from the interconnection between the plant in (2) and the hybrid observer in (14), which is given in (15), has the following properties:

(1) It is well-posed;

(2) For each initial condition in $\mathcal{C} \cup \mathcal{D}$, there exists a maximal solution. In addition, each of its maximal solutions is complete.

Furthermore, for any Lebesgue measurable inputs $t \mapsto$ $u(t)$ and $t \mapsto w(t)$ defined on $[0, \infty)$, every maximal solution to (15) is complete. 
Proof. According to [20], when $u \equiv 0$ and $w \equiv 0$, the data of the hybrid system in (15) is given by

$$
F(x, \hat{x}, q)=\left[\begin{array}{c}
A x \\
A \hat{x}+L_{q}(C x-C \hat{x}) \\
0
\end{array}\right]
$$

for all $(x, \hat{x}, q)$ such that $(\hat{x}, q, C x, 0,0) \in \mathcal{C}$, and

$$
G(x, \hat{x}, q)=\left[\begin{array}{c}
x \\
\hat{x} \\
3-q
\end{array}\right]
$$

for all $(x, \hat{x}, q)$ such that $(\hat{x}, q, C x, 0,0) \in \mathcal{D}$. By construction, the sets $\mathcal{C}$ and $\mathcal{D}$ are closed since they are the union of closed sets. The maps $F$ and $G$ are linear in the state. Then, since $(\mathcal{C}, F, \mathcal{D}, G)$ satisfies the hybrid basic conditions in [20, Assumption 6.5, p. 120], (15) is wellposed for zero inputs.

Next, we show that every maximal solution with zero inputs is complete. Let $(x, \hat{x}, q)$ be such that $(\hat{x}, q, C x, 0,0) \in \mathcal{C} \backslash \mathcal{D}$. If $q=1$, then $|C x-C \hat{x}|>\delta_{1} \theta_{\text {th }}$, and since $q$ remains constant during flows and $F$ is linear in the state, flow of (15) is possible for a nonzero finite amount of time. Hence, condition (VC) in [20, Proposition 2.10, p. 33] holds - and, consequently, (VC) in [20, Proposition 6.10, p. 124] holds. If $q=2$, then $|C x-C \hat{x}|<\delta_{2} \theta_{\mathrm{th}}$, and flow is possible for a nonzero finite amount of time. As a consequence, via [20, Proposition 6.10, p. 124], there exists a nontrivial solution from every point in $\mathcal{C} \cup \mathcal{D}$; that is, there exists a solution that either flows for some nonzero finite amount of time or jumps at least once. To show that every maximal solution is complete, we show that (b) and (c) in $\left[20\right.$, Proposition 6.10, p. 124] do not hold. Due to $\mathcal{C}_{q}$ and $\mathcal{D}_{q}$ being, for each $q \in\{1,2\}$, the closed complement of each other (with the same value of $q$ ), by definition of $G$, every point in $\mathcal{C} \cup \mathcal{D}$ is mapped by $G$ to a point in $\mathcal{C} \cup \mathcal{D}$. Then, (c) does not hold. Condition (b) does not hold due to linearity of $F$. Then, by [20, Proposition 6.10 , p. 124], every maximal solution to (15) with zero inputs is complete.

Finally, when the inputs are not necessarily equal to zero but Lebesgue measurable, completeness of maximal solutions can be established via [16, Proposition 3.4, p. 2429].

The QB of the estimation error holds as follows.

Theorem 2 If there exist a symmetric matrix $P>0$, $Y_{1}, Y_{2} \in \mathbb{R}^{n \times m}, \alpha_{1}, \alpha_{2} \in \mathbb{R}_{>0}^{s}$, and scalars $\beta_{1}, \beta_{2}, \gamma_{1}, \gamma_{2}>$
$0, \delta_{1}, \delta_{2} \in(0,1)$ such that

$$
\begin{gathered}
\left(\begin{array}{cc}
A^{\top} P-C^{\top} Y_{1}+P A-Y_{1} C & P D-Y_{1} E \\
+\beta_{1} P+\gamma_{1} C^{\top} C & +\gamma_{1} C^{\top} E \\
\star & \gamma_{1} E^{\top} E-\operatorname{diag}\left(\alpha_{1}\right)
\end{array}\right)<0 \\
\left(\begin{array}{cc}
A^{\top} P-C^{\top} Y_{2}+P A-Y_{2} C & P D-Y_{2} E \\
+\beta_{2} P-\gamma_{2} C^{\top} C & -\gamma_{2} C^{\top} E \\
\star & -\gamma_{2} E^{\top} E-\operatorname{diag}\left(\alpha_{2}\right)
\end{array}\right)<0 \\
\\
1^{\top} \alpha_{1}-\beta_{1}-\gamma_{1} \delta_{1}^{2} \theta_{\mathrm{th}}^{2} \leq 0 \\
1^{\top} \alpha_{2}-\beta_{2}+\gamma_{2} \delta_{2}^{2} \theta_{\mathrm{th}}^{2} \leq 0
\end{gathered}
$$

hold, then the estimation error dynamics resulting from the observer (14) with gains $L_{1}=P^{-1} Y_{1}$ and $L_{2}=$ $P^{-1} Y_{2}$ is quadratically bounded with $V(e)=e^{\top} P e$, see (6).

Proof. From (2) and (14) it follows that

$$
\begin{aligned}
& \left.\begin{array}{l}
\dot{e}=\left(A-L_{q} C\right) e+\left(D-L_{q} E\right) w \\
\dot{q}=0
\end{array}\right\}(e, q, w) \in \tilde{\mathcal{C}} \\
& e^{+}=e \\
& q^{+}=3-q \\
& \}(e, q, w) \in \tilde{\mathcal{D}}
\end{aligned}
$$

where $\tilde{\mathcal{C}}=\tilde{\mathcal{C}}_{1} \cup \tilde{\mathcal{C}}_{2}$ and $\tilde{\mathcal{D}}=\tilde{\mathcal{D}}_{1} \cup \tilde{\mathcal{D}}_{2}$ with

$$
\begin{aligned}
& \tilde{\mathcal{C}_{1}}:=\left\{(e, q, w) \in \mathbb{R}^{n} \times\{1,2\} \times[-1,1]^{m}:|C e+E w|\right. \\
& \left.\geq \delta_{1} \theta_{\mathrm{th}}, q=1\right\} \text {, } \\
& \tilde{\mathcal{D}}_{1}:=\left\{(e, q, w) \in \mathbb{R}^{n} \times\{1,2\} \times[-1,1]^{m}:|C e+E w|\right. \\
& \left.\leq \delta_{1} \theta_{\mathrm{th}}, q=1\right\}, \\
& \tilde{\mathcal{C}_{2}}:=\left\{(e, q, w) \in \mathbb{R}^{n} \times\{1,2\} \times[-1,1]^{m}:|C e+E w|\right. \\
& \left.\leq \delta_{2} \theta_{\mathrm{th}}, q=2\right\}, \\
& \tilde{\mathcal{D}}_{2}:=\left\{(e, q, w) \in \mathbb{R}^{n} \times\{1,2\} \times[-1,1]^{m}:|C e+E w|\right. \\
& \left.\geq \delta_{2} \theta_{\mathrm{th}}, q=2\right\} \text {. }
\end{aligned}
$$

If $(e, q, w) \in \tilde{\mathcal{C}}_{1}$, we need to account for $|C e+E w| \geq$ $\delta_{1} \theta_{\mathrm{th}}$, while we have $|C e+E w| \leq \delta_{2} \theta_{\mathrm{th}}$ if $(e, q, w) \in \tilde{\mathcal{C}}_{2}$. Thus, since $\tilde{\mathcal{C}}=\tilde{\mathcal{C}}_{1} \cup \tilde{\mathcal{C}}_{2}$, it follows that the time derivative of the Lyapunov function $V(e)=e^{\top} P e$ is given by

$$
\begin{aligned}
& \dot{V}(e, q, w)=e^{\top}\left(\left(A-L_{q} C\right)^{\top} P+P\left(A-L_{q} C\right)\right) e \\
& +w^{\top}\left(D^{\top}-E^{\top} L_{q}^{\top}\right) P e+e^{\top} P\left(D-L_{q} E\right) w
\end{aligned}
$$

for each $(e, q, w) \in \tilde{\mathcal{C}}$. From now on, using $Y_{q}=P L_{q} \in$ 
$\mathbb{R}^{n \times m}$ with $q \in\{1,2\}$, consider the equality

$$
\begin{aligned}
& e^{\top}\left(\left(A-L_{q} C\right)^{\top} P+P\left(A-L_{q} C\right)\right) e \\
& +w^{\top}\left(D^{\top}-E^{\top} L_{q}^{\top}\right) P e+e^{\top} P\left(D-L_{q} E\right) w \\
& =e^{\top}\left(A^{\top} P-C^{\top} Y_{q}^{\top}+P A-Y_{q} C\right) e \\
& +w^{\top}\left(D^{\top} P-E^{\top} Y_{q}^{\top}\right) e+e^{\top}\left(P D-Y_{q} E\right) w .
\end{aligned}
$$

If $q=1$, we need to account for the condition $\mid C e+$ $E w \mid \geq \delta_{1} \theta_{\mathrm{th}}$, which squaring both sides leads to

$$
\begin{aligned}
& -e^{\top} C^{\top} C e-e^{\top} C^{\top} E w-w^{\top} E^{\top} C e-w^{\top} E^{\top} E w \\
& +\delta_{1}^{2} \theta_{\mathrm{th}}^{2} \leq 0 .
\end{aligned}
$$

If $q=2$, from $|C e+E w| \leq \delta_{2} \theta_{\text {th }}$ it follows that

$$
\begin{aligned}
& e^{\top} C^{\top} C e+e^{\top} C^{\top} E w+w^{\top} E^{\top} C e+w^{\top} E^{\top} E w \\
& -\delta_{2}^{2} \theta_{\text {th }}^{2} \leq 0 .
\end{aligned}
$$

Thus, we have to impose $\dot{V}(e, q, w)<0$ with $(22)$ when $q=1$ and (23) when $q=2$ for all $e \in \mathbb{R}^{n}$ and $w_{i} \in \mathbb{R}$ such that

$$
-e^{\top} P e+1<0
$$

and

$$
w_{i}^{2}-1 \leq 0, i=1, \ldots, s .
$$

Using the S-procedure (see, e.g., [14, p. 23]), it is straightforward to impose that the time derivative of the Lyapunov function $V(e)$ by accounting for switching condition (22) and (23) with (24), and (25) by means of scalar parameters that, for the sake of brevity, are lumped in $\alpha_{1}, \alpha_{2} \in \mathbb{R}_{>0}^{s}$ and $\beta_{1}, \beta_{2}, \gamma_{1}, \gamma_{2}>0$. More specifically, a sufficient condition for $\dot{V}(e, q, w)<0$ to hold subject to $(22),(23),(24)$, and (25) is given by the existence of $\alpha_{1} \in \mathbb{R}_{>0}^{s}$ and $\beta_{1}, \gamma_{1}>0$ such that

$$
\begin{aligned}
& e^{\top}\left(A^{\top} P-C^{\top} Y_{1}^{\top}+P A-Y_{1} C+\beta_{1} P+\gamma_{1} C^{\top} C\right) e \\
& +w^{\top}\left(D^{\top} P-E^{\top} Y_{1}^{\top}+\gamma_{1} E^{\top} C\right) e+e^{\top}(P D \\
& \left.-Y_{1} E+\gamma_{1} C^{\top} E\right) w+w^{\top}\left(\gamma_{1} E^{\top} E-\operatorname{diag}\left(\alpha_{1}\right)\right) w \\
& +1^{\top} \alpha_{1}-\beta_{1}-\gamma_{1} \delta_{1}^{2} \theta_{\mathrm{th}}^{2} \leq 0
\end{aligned}
$$

and $\alpha_{2} \in \mathbb{R}_{>0}^{s}$ and $\beta_{2}, \gamma_{2}>0$ such that

$$
\begin{aligned}
& e^{\top}\left(A^{\top} P-C^{\top} Y_{2}^{\top}+P A-Y_{2} C+\beta_{2} P-\gamma_{2} C^{\top} C\right) e \\
& +w^{\top}\left(D^{\top} P-E^{\top} Y_{2}^{\top}-\gamma_{2} E^{\top} C\right) e+e^{\top}(P D \\
& \left.-Y_{2} E-\gamma_{2} C^{\top} E\right) w+w^{\top}\left(-\gamma_{2} E^{\top} E-\operatorname{diag}\left(\alpha_{2}\right)\right) w \\
& +1^{\top} \alpha_{2}-\beta_{2}+\gamma_{2} \delta_{2}^{2} \theta_{\mathrm{th}}^{2} \leq 0 .
\end{aligned}
$$

Such conditions are satisfied if (16), (17), (18), (19) together with (20) hold. In addition, note that the Lyapunov function does not change in case of jumps since from (21b) it follows that $V\left(e^{+}\right)=V(e)$. Then, since the Lyapunov function does not increase during jumps of (21), the estimation error is quadratically bounded.
Notice that the stability conditions of Theorem 2 are BMIs. The design of the observer (14) results from the solution of the optimization problem

$$
\begin{aligned}
& \max _{P>0 ; Y_{1}, Y_{2} ; \alpha_{1}, \alpha_{2} \in \mathbb{R}_{>0}^{s} ; \beta_{1}, \beta_{2}, \gamma_{1}, \gamma_{2}, \lambda>0 ; \delta_{1}, \delta_{2} \in(0,1)} \lambda \\
& \text { s.t. } P>\lambda I,(16),(17),(18),(19),(20) .
\end{aligned}
$$

Remark 2 A generalization of the considered switchedgain observer consists in adopting a multi-gain scheduling given by (14) with a number of gains equal to $k \geq 3$ by using

$$
\mathcal{C}=\bigcup_{i=1}^{k} \mathcal{C}_{i} \quad \mathcal{D}=\bigcup_{i=1}^{k} \mathcal{D}_{i}
$$

with

$$
\begin{array}{r}
\mathcal{C}_{i}:=\left\{(\hat{x}, q, y, u, w) \in \mathbb{R}^{n} \times\{1, \ldots, k\} \times \mathbb{R}^{m} \times \mathbb{R}^{p}\right. \\
\left.\times[-1,1]^{s}:|y-C \hat{x}| \geq \delta_{i} \theta_{\mathrm{th}}, q=i\right\}, \\
\mathcal{D}_{i}:=\left\{(\hat{x}, q, y, u, w) \in \mathbb{R}^{n} \times\{1, \ldots, k\} \times \mathbb{R}^{m} \times \mathbb{R}^{p}\right. \\
\left.\times[-1,1]^{s}:|y-C \hat{x}| \leq \delta_{i} \theta_{\mathrm{th}}, q=i\right\},
\end{array}
$$

for all odd integer $i \leq k$,

$$
\begin{array}{r}
\mathcal{C}_{l}:=\left\{(\hat{x}, q, y, u, w) \in \mathbb{R}^{n} \times\{1, \ldots, k\} \times \mathbb{R}^{m} \times \mathbb{R}^{p}\right. \\
\left.\times[-1,1]^{s}:|y-C \hat{x}| \leq \delta_{l} \theta_{\mathrm{th}}, q=l\right\}, \\
\mathcal{D}_{l}:=\left\{(\hat{x}, q, y, u, w) \in \mathbb{R}^{n} \times\{1, \ldots, k\} \times \mathbb{R}^{m} \times \mathbb{R}^{p}\right. \\
\left.\times[-1,1]^{s}:|y-C \hat{x}| \geq \delta_{l} \theta_{\mathrm{th}}, q=l\right\},
\end{array}
$$

for all even integer $l \leq k$, where $\delta_{i} \in(0,1), i=1, \ldots, k$, such that $\delta_{i}<\delta_{i+1}, i=1, \ldots, k-1$, and gains $L_{1}, \ldots, L_{k} \in \mathbb{R}^{n \times m}$.

Since (14) may undergo a large number of consecutive jumps, the switching can be regularized by imposing a hold time, i.e., a minimum dwell time required to keep the observer with the same gain. Thus, consider the hybrid observer

$$
\begin{aligned}
& \left.\begin{array}{l}
\dot{\hat{x}}=A \hat{x}+B u+L_{q}(y-C \hat{x}) \\
\dot{q}=0 \\
\dot{\tau}=2-q
\end{array}\right\}(\hat{x}, \tau, q, y, u, w) \in \mathcal{C} \\
& \hat{x}^{+}=\hat{x} \\
& q^{+}=3-q \\
& \tau^{+}=0
\end{aligned}
$$

where $y=C x+E w, \mathcal{C}=\mathcal{C}_{1} \cup \mathcal{C}_{2}, \mathcal{D}=\mathcal{D}_{1} \cup \mathcal{D}_{2}$ with

$$
\begin{aligned}
\mathcal{C}_{1}:= & \left\{(\hat{x}, \tau, q, y, u, w) \in \mathbb{R}^{n} \times \mathbb{R}_{\geq 0} \times\{1,2\} \times \mathbb{R}^{m} \times \mathbb{R}^{p}\right. \\
& \left.\times[-1,1]^{s}:|y-C \hat{x}| \geq \delta_{1} \theta_{\mathrm{th}} \text { or } \tau \in\left[0, T_{h}\right], q=1\right\}, \\
\mathcal{C}_{2}:= & \left\{(\hat{x}, \tau, q, y, u, w) \in \mathbb{R}^{n} \times \mathbb{R}_{\geq 0} \times\{1,2\} \times \mathbb{R}^{m} \times \mathbb{R}^{p}\right.
\end{aligned}
$$


$\left.\times[-1,1]^{s}:|y-C \hat{x}| \leq \delta_{2} \theta_{\mathrm{th}}, \tau=0, q=2\right\}$,

$\mathcal{D}_{1}:=\left\{(\hat{x}, \tau, q, y, u, w) \in \mathbb{R}^{n} \times \mathbb{R}_{\geq 0} \times\{1,2\} \times \mathbb{R}^{m} \times \mathbb{R}^{p}\right.$ $\left.\times[-1,1]^{s}:|y-C \hat{x}| \leq \delta_{1} \theta_{\mathrm{th}}, \tau=T_{h}, q=1\right\}$,

$\mathcal{D}_{2}:=\left\{(\hat{x}, \tau, q, y, u, w) \in \mathbb{R}^{n} \times \mathbb{R}_{\geq 0} \times\{1,2\} \times \mathbb{R}^{m} \times \mathbb{R}^{p}\right.$ $\left.\times[-1,1]^{s}:|y-C \hat{x}| \geq \delta_{2} \theta_{\mathrm{th}}, q=2\right\}$,

$\delta_{1}, \delta_{2} \in(0,1), \delta_{1}<\delta_{2}$, and hold time $T_{h}>0$.

Following a reasoning like in the proof of Proposition 1, the solution of the switched-gain observer (29) can be proved to be complete for any $\hat{x}(0) \in \mathbb{R}^{n}$.

For the observer (29), similar conditions to those presented for (14) are established, as follows.

Theorem 3 If there exist a symmetric matrix $P>0$, $Y_{1}, Y_{2} \in \mathbb{R}^{n \times m}, \alpha_{1}, \alpha_{2} \in \mathbb{R}_{>0}^{s}$, and scalars $\beta_{1}, \beta_{2}, \gamma>0$, $\delta_{1}, \delta_{2} \in(0,1)$ such that

$$
\begin{aligned}
& \left(\begin{array}{cc}
A^{\top} P-C^{\top} Y_{1}+P A-Y_{1} C+\beta_{1} P & P D-Y_{1} E \\
\star & -\operatorname{diag}\left(\alpha_{1}\right)
\end{array}\right)<0 \\
& \left(\begin{array}{cc}
A^{\top} P-C^{\top} Y_{2}+P A-Y_{2} C & P D-Y_{2} E \\
+\beta_{2} P-\gamma C^{\top} C & -\gamma C^{\top} E \\
\star & -\gamma E^{\top} E-\operatorname{diag}\left(\alpha_{2}\right)
\end{array}\right)<0 \\
& (30) \\
& 1^{\top} \alpha_{1}-\beta_{1} \leq 0 \\
& 1^{\top} \alpha_{2}-\beta_{2}+\gamma \delta_{2}^{2} \theta_{\text {th }}^{2} \leq 0 \\
& \delta_{1}<\delta_{2}
\end{aligned}
$$

hold, then the estimation error dynamics resulting from the observer (29) with gains $L_{1}=P^{-1} Y_{1}$ and $L_{2}=$ $P^{-1} Y_{2}$ is quadratically bounded with $V(e)=e^{\top} P e$.

Proof. In line with the proof of Theorem 2, notice that the condition $|r|=|y-C \hat{x}| \geq \delta_{1} \theta_{\text {th }}$ might not be satisfied if $(\hat{x}, \tau, q, y, u, w) \in \mathcal{C}_{1}$ during the hold time. Instead, we have still $|r|=|y-C \hat{x}| \leq \delta_{2} \theta_{\mathrm{th}}$ if $(\hat{x}, \tau, q, y, u, w) \in$ $\mathcal{C}_{2}$. Thus, we have to account only for (24) and (25) if $q=1$ and (23) together with (24) and (25) if $q=2$. This allows to conclude by following the same steps of the proof of Theorem 2 .

The design of the observer (29) can be done by solving

$$
\begin{aligned}
& \max _{P>0 ; Y_{1}, Y_{2} ; \alpha_{1}, \alpha_{2} \in \mathbb{R}_{>0}^{s} ; \beta_{1}, \beta_{2}, \gamma, \lambda>0 ; \delta_{2} \in(0,1)} \lambda \\
& \text { s.t. } P>\lambda I,(30),(31),(32),(33) .
\end{aligned}
$$

Notice that the choice of $\delta_{1}$ needs to be done after solving
(35) by choosing this parameter strictly less than the value of $\delta_{2}$ resulting from the solution of (35).

In the next section, we will show numerical results concerning what proposed so far.

\section{Simulation Results}

Two case studies were addressed as shown in Sections 4.1 and 4.2 , where the results of the design procedures and simulation tests are described. The simulations were obtained by using the Hybrid Equations Toolbox [31].

\subsection{Oscillator with a Measurement Bias}

Let us consider an autonomous oscillating system with a measurement bias affecting the output and disturbances with modulus no higher than 0.1 , i.e.,

$$
A=\left(\begin{array}{rrr}
0 & 1 & 0 \\
-1 & 0 & 0 \\
0 & 0 & 0
\end{array}\right) \quad C=\left(\begin{array}{lll}
1 & 0 & 1
\end{array}\right) \quad D=\left(\begin{array}{rr}
0 & 0 \\
0 & 0.1 \\
0 & 0
\end{array}\right)
$$

and $E=\left(\begin{array}{ll}0.1 & 0\end{array}\right)$.

For the purpose of comparison, we constructed the observer (3) by solving (12), thus providing

$$
\begin{aligned}
& P=\left(\begin{array}{rrr}
13.9358 & -4.5095 & 8.5645 \\
-4.5095 & 9.7753 & -9.2828 \\
8.5645 & -9.2828 & 29.9254
\end{array}\right) \quad L=\left(\begin{array}{l}
1.0544 \\
0.9370 \\
0.4745
\end{array}\right) \\
& \alpha=\left(\begin{array}{l}
0.1453 \\
0.1578
\end{array}\right) \quad \beta=0.3031
\end{aligned}
$$

and hence $\rho_{e}=0.4117, \theta_{\mathrm{th}}=0.6822$; the value of $\lambda$ is just equal to the minimum eigenvalue of $P$ and therefore is omitted here and in what follows for the sake of brevity. We will refer to this estimator as QB observer or QBO, for short. The design of the QBO was obtained by using a simple bisection method first to find the maximum $\beta>0$ and then apply a search method for maximizing the minimum eigenvalue of $P$, while eventually reduce $\beta$ as well as selecting the remaining parameters (see, e.g., $[2,4]$ for similar approaches). By contrast, switchedgain observers require to deal with more complex BMIs. Specifically, the solution of the optimization problems involved in the design of such observers to maximize the minimum eigenvalue of $P$ was obtained by using the generalized pattern search (GPS) algorithm, which consists in performing a local search on a grid around the current point in such a way as to reduce the cost at each iteration [13]. This search was carried out by using the Matlab routine patternsearch to select some parameters 
while solving the LMI-based design conditions by means of YALMIP [24] to find the remaining parameters.

We solved (28) to construct the observer (14) and found

$P=\left(\begin{array}{rrr}17.4271 & -5.7903 & 11.8792 \\ -5.7903 & 11.6919 & -11.9934 \\ 11.8792 & -11.9934 & 32.4385\end{array}\right) \quad L_{1}=\left(\begin{array}{l}0.8691 \\ 1.1827 \\ 0.4476\end{array}\right)$

$L_{2}=\left(\begin{array}{l}1.1151 \\ 1.2468 \\ 0.6771\end{array}\right) \quad \alpha_{1}=\left(\begin{array}{l}0.2202 \\ 0.1907\end{array}\right) \quad \alpha_{2}=\left(\begin{array}{l}0.2026 \\ 0.1983\end{array}\right)$

$\beta_{1}=0.1076 \quad \beta_{2}=0.4009 \quad \gamma_{1}=0.9414$

$\gamma_{2}=1.0 \mathrm{e}-14 \quad \delta_{1}=0.8935 \quad \delta_{2}=0.9482$

with $\rho_{e}=0.4019$. We will refer to this observer as switched-gain QB observer (SGQBO).

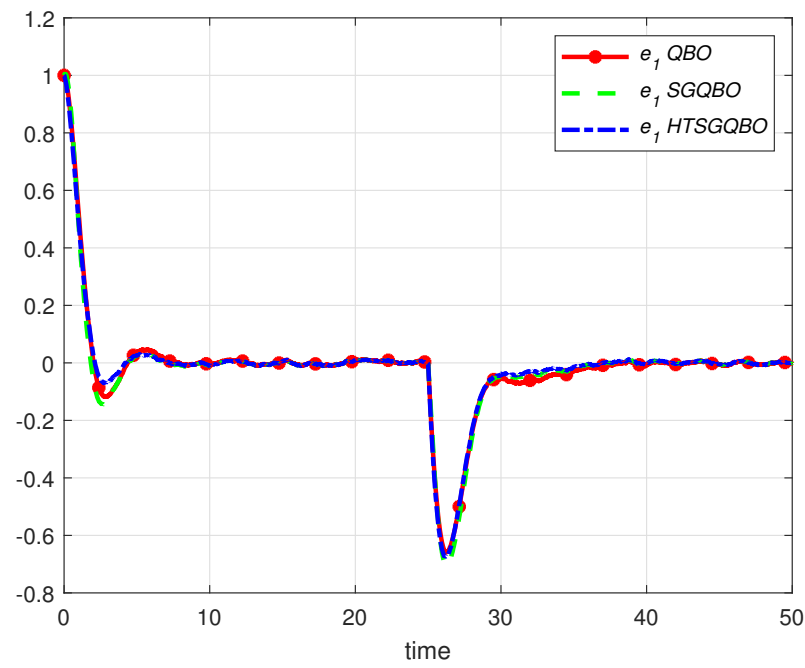

Fig. 2. Evolution of the estimation errors in the first state variable.

We solved (35) to find the gains of (29) and $\delta_{2}$; the corresponding estimator will be denoted as hold-time switched-gain QB observer (HTSGQBO). We obtained the following results:

$P=\left(\begin{array}{rrr}17.4265 & -5.7901 & 11.8786 \\ -5.7901 & 11.6917 & -11.9930 \\ 11.8786 & -11.9930 & 32.4379\end{array}\right) L_{1}=\left(\begin{array}{l}1.1151 \\ 1.2468 \\ 0.6771\end{array}\right)$

$L_{2}=\left(\begin{array}{l}1.1151 \\ 1.2468 \\ 0.6771\end{array}\right) \quad \alpha_{1}=\left(\begin{array}{l}0.2026 \\ 0.1983\end{array}\right) \quad \alpha_{2}=\left(\begin{array}{l}0.2026 \\ 0.1983\end{array}\right)$

$\beta_{1}=0.4009 \quad \beta_{2}=0.4009 \quad \gamma=1.074 \mathrm{e}-10 \quad \delta_{2}=0.4658$

and chose $T_{h}=3$ and $\delta_{1}=\delta_{2} / 2$ for the HTSGQBO $\left(\rho_{e}=0.4019\right)$. Notice that the gains $L_{1}, L_{2}$ coincide,

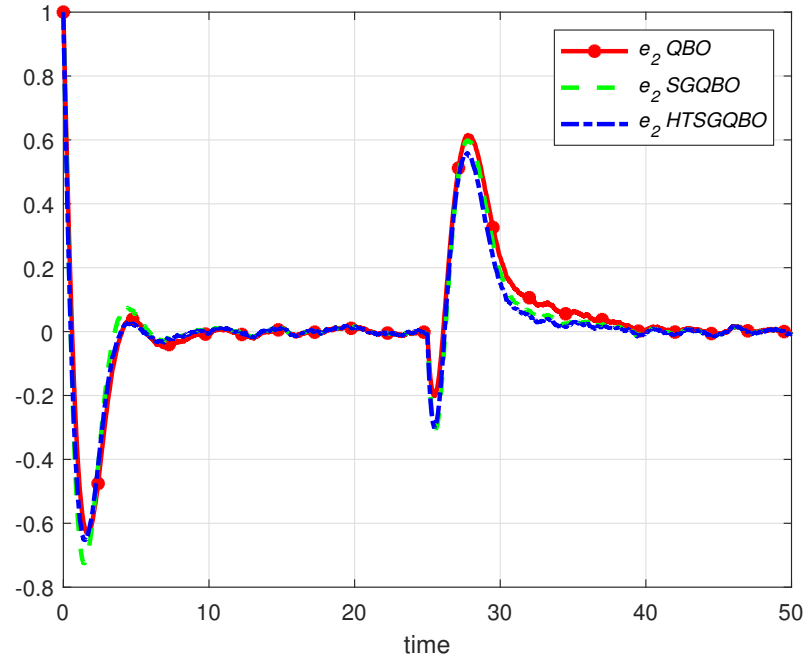

Fig. 3. Evolution of the estimation errors in the second state variable.

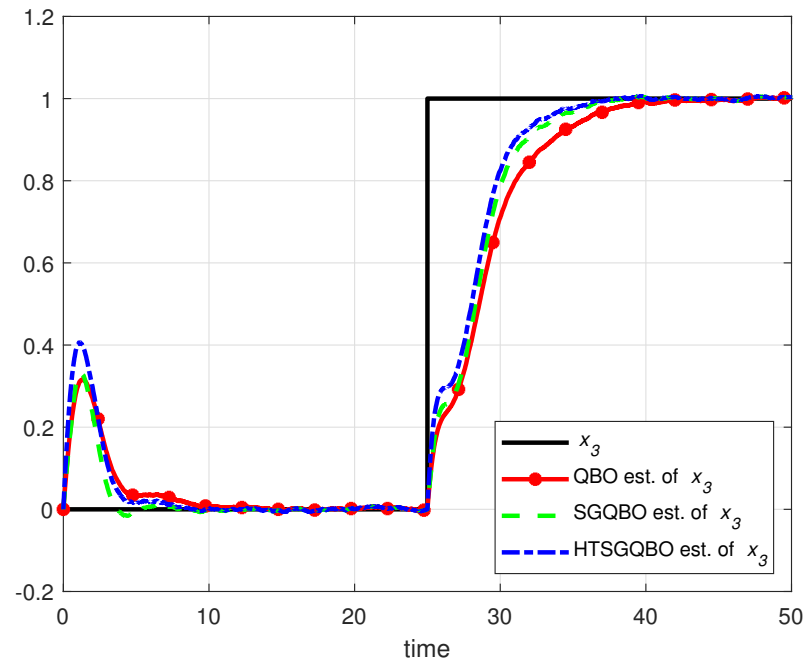

Fig. 4. Evolution of $x_{3}$ and its estimates.

which suggests the investigation of observers based on Lyapunov functions with a richer structure such as piecewise quadratic Lyapunov functions (see, among others, $[21])$.

Table 1 reports the different asymptotic bounds, thus highlighting the effectiveness of the proposed optimization-based design for SGQBO and HTSGQBO as compared to QBO. Indeed, the HTSGQBO design provided the same gains but the hold time setup turns out really effective in the transient, as shown in Figs. $2-6$, where the results of a simulation run with initial state $(0.5,0.5,0)$, estimated initial states all equal to $(-0.5,-0.5,0)$, disturbances chosen according to a uniform distribution in $[-1,1]$, and a unitary bias on the output occurring at time $t=25 \mathrm{~s}$ as a step function are presented.

As shown in Figs. 2-4, the QBO performs worse than the 


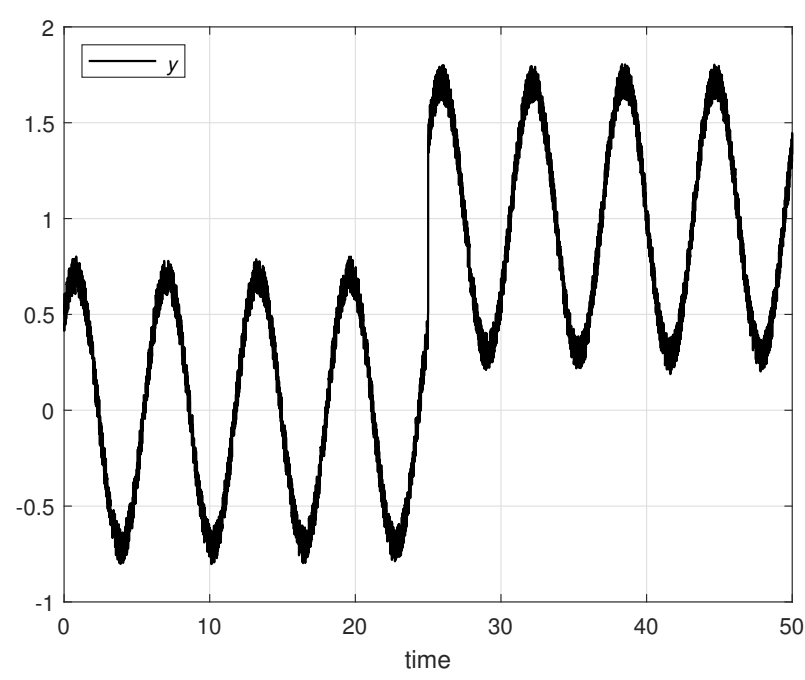

Fig. 5. Evolution of $y$.
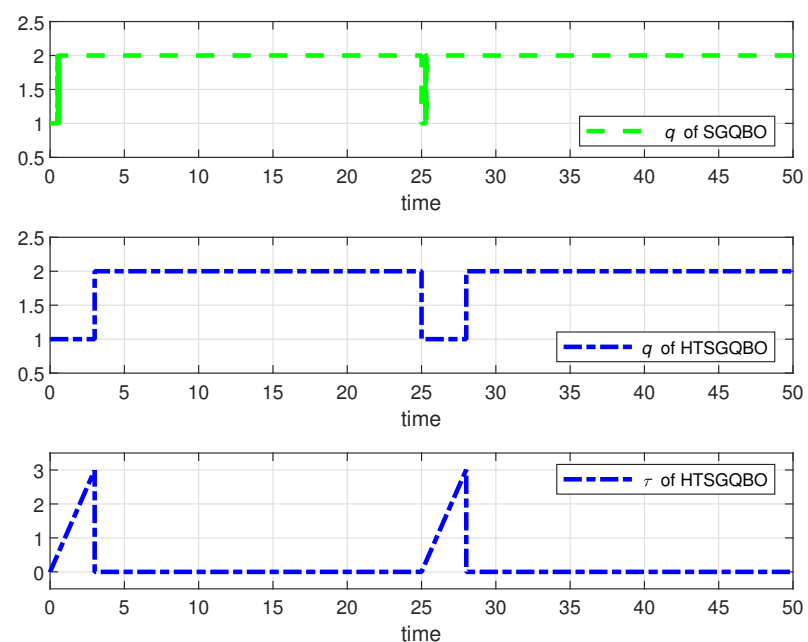

Fig. 6. Evolution of $q$ given by the SGQBO and of $q, \tau$ given by the HTSGQBO.

Table 1

Steady-state asymptotic bounds

\begin{tabular}{cccc}
\hline & QBO & SGQBO & HTSGQBO \\
\hline$\rho_{e}$ & 0.4117 & 0.4019 & 0.4019 \\
\hline
\end{tabular}

SGQBO and HTSGQBO with lower rate of convergence (see, e.g., in Fig. 4 the slow adaptation of the QBO bias estimate to the true value).

\subsection{Unstable System with a Measurement Bias}

Let us consider an unstable system with output subject to a bias likewise in Section 4.1, i.e., with the very same matrices except $A$ given by

$$
A=\left(\begin{array}{rrr}
0 & 1 & 0 \\
-1 & 1 & 0 \\
0 & 0 & 0
\end{array}\right)
$$

We designed the observer (3) by solving (12), thus obtaining

$$
\begin{gathered}
P=\left(\begin{array}{rrr}
21.4919 & -8.2726 & 23.9463 \\
-8.2726 & 5.6839 & -12.5315 \\
23.9463 & -12.5315 & 42.4601
\end{array}\right) L=\left(\begin{array}{l}
2.6823 \\
6.3306 \\
1.1007
\end{array}\right) \\
\alpha=\left(\begin{array}{l}
0.3163 \\
0.1197
\end{array}\right) \beta=0.4360 \rho_{e}=0.76986 \theta_{\mathrm{th}}=1.1887
\end{gathered}
$$

for the QBO. Concerning the SGQBO, we solved (28) and got

$$
\begin{aligned}
& P=\left(\begin{array}{rrr}
27.1638 & -10.1011 & 30.4564 \\
-10.1011 & 6.6698 & -15.0113 \\
30.4564 & -15.0113 & 47.6395
\end{array}\right) \quad L_{1}=\left(\begin{array}{l}
2.3891 \\
7.4225 \\
1.6145
\end{array}\right) \\
& L_{2}=\left(\begin{array}{l}
2.5161 \\
7.6372 \\
1.7003
\end{array}\right) \quad \alpha_{1}=\left(\begin{array}{l}
0.43708 \\
0.16105
\end{array}\right) \quad \alpha_{2}=\left(\begin{array}{l}
0.42987 \\
0.15328
\end{array}\right) \\
& \beta_{1}=\beta_{2}=0.5981 \gamma_{1}=0.375 \gamma_{2}=0.25 \delta_{1}=0.14142 \\
& \delta_{2}=0.17321 \quad \rho_{e}=0.75482 .
\end{aligned}
$$

We solved (35) to design an HTSGQBO, for which we chose $T_{h}=3$. We obtained

$P=\left(\begin{array}{rrr}27.9835 & -10.3860 & 31.3830 \\ -10.3860 & 6.8430 & -15.4179 \\ 31.3830 & -15.4179 & 48.8230\end{array}\right) \quad L_{1}=\left(\begin{array}{l}2.5029 \\ 7.7147 \\ 1.7380\end{array}\right)$

$L_{2}=\left(\begin{array}{l}2.5029 \\ 7.7147 \\ 1.7380\end{array}\right) \quad \alpha_{1}=\left(\begin{array}{r}0.4446 \\ 0.15813\end{array}\right) \quad \alpha_{2}=\left(\begin{array}{r}0.4446 \\ 0.15813\end{array}\right)$

$\beta_{1}=\beta_{2}=0.60273 \gamma=8.7817 \mathrm{e}-11 \delta_{2}=0.96986$

and chose $\delta_{1}=0.4849$. The asymptotic bounds obtained for QBO, SGQBO, and HTSGQBO are shown in Table 2. The results of a simulation run with initial state $(0.5,0.5,0)$, estimated initial states all equal to $(-0.5,-0.5,0)$, disturbances chosen according to a uniform distribution in $[-1,1]$, and a bias equal to 2 on the output occurring at time $t=25 \mathrm{~s}$ are presented in Figs. 7-11. Again, SGQBO and especially HTSGQBO turn out to perform much better than QBO. 
Table 2

Steady-state asymptotic bounds

\begin{tabular}{cccc}
\hline & QBO & SGQBO & HTSGQBO \\
\hline$\rho_{e}$ & 0.76986 & 0.75482 & 0.74800 \\
\hline
\end{tabular}

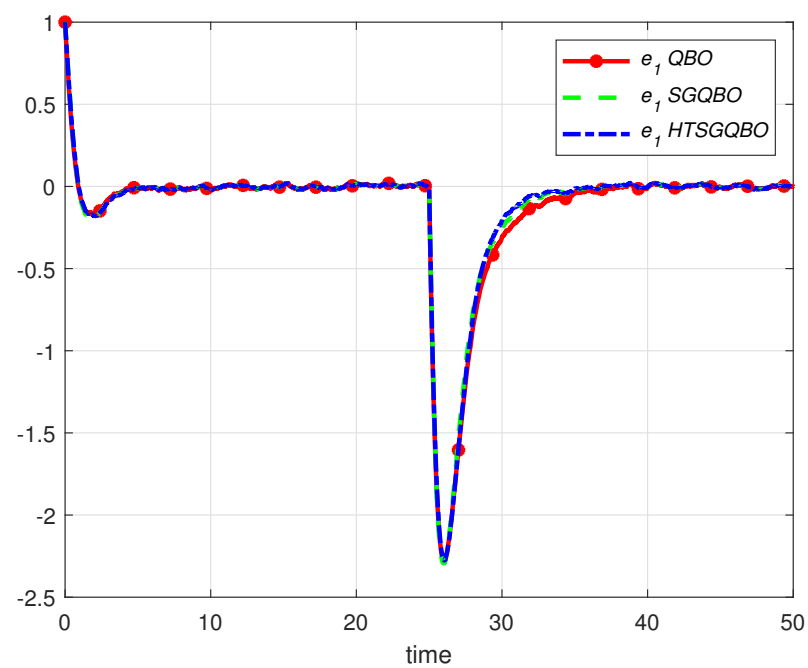

Fig. 7. Evolution of the estimation errors in the first state variable.

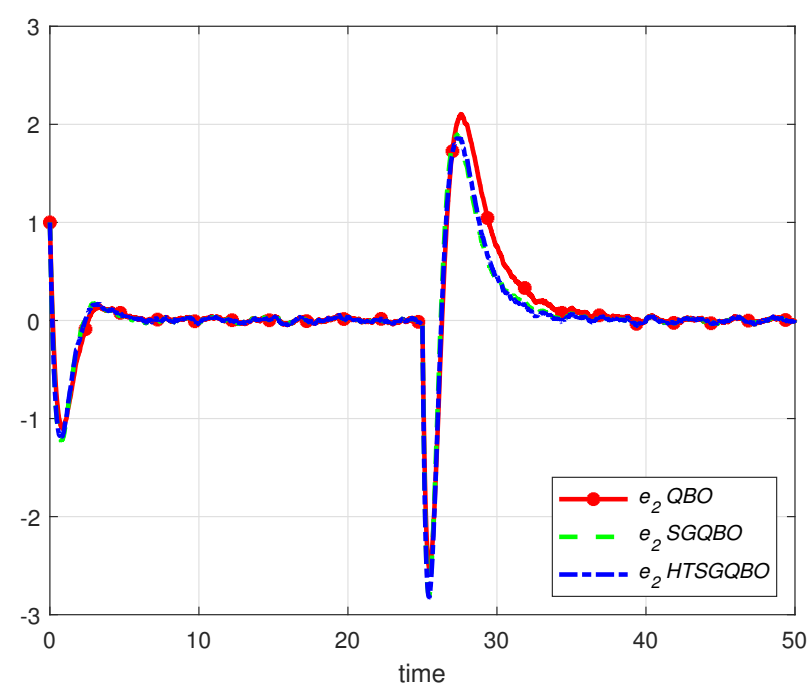

Fig. 8. Evolution of the estimation errors in the second state variable.

\section{Conclusion}

Hysteresis-based switching observers for linear systems affected by bounded disturbances have been studied in the hybrid systems framework by relying on QB and investigating the use of a hold time mechanism to increase the performances in the transient. Future work will concern piecewise quadratic Lyapunov functions to construct more effective observers by taking advantage of their richer structure with additional parameters to be tuned as compared with standard quadratic Lyapunov

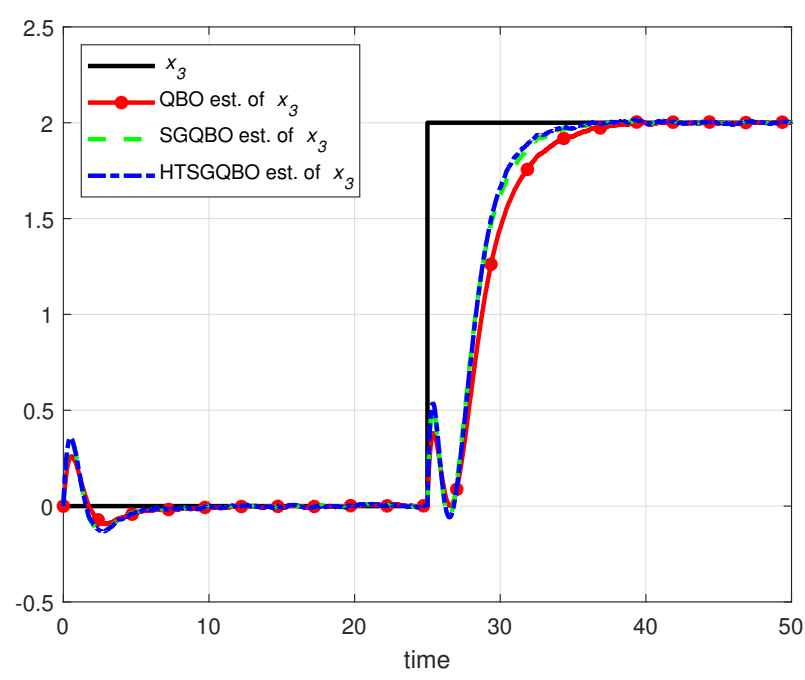

Fig. 9. Evolution of $x_{3}$ and its estimates.

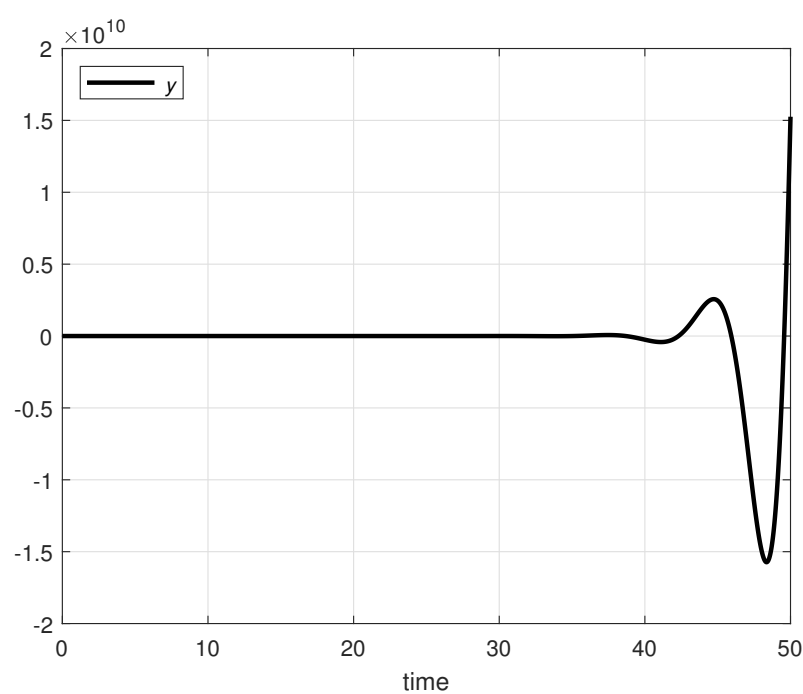

Fig. 10. Evolution of $y$.

functions. The extension of the proposed approach to deal with systems with Lipschitz nonlinearities by using, for example, high-gain observers [1] will be addressed as well. Another topic of interest is estimation for switching systems, while accounting for sensor and actuator faults and exploiting bounds on the estimation error for a consistent choice of the detection threshold.

\section{Acknowledgment}

The authors would like to thank Prof. Franco Blanchini for her helpful comments and suggestions.

\section{References}

[1] J. H. Ahrens and H. K. Khalil. High-gain observers in the presence of measurement noise: A switched-gain approach. Automatica, 45(4):936 - 943, 2009. 

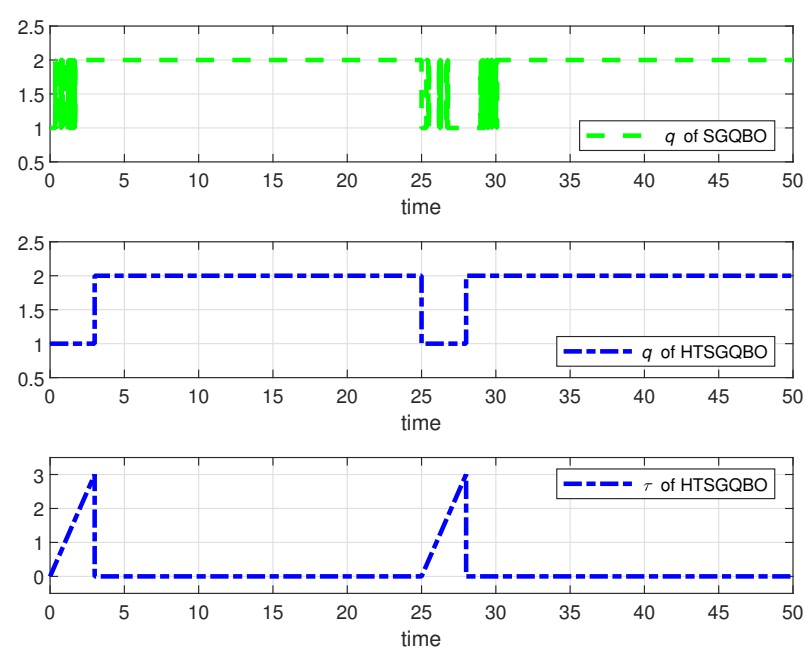

Fig. 11. Evolution of $q$ given by the SGQBO and of $q, \tau$ given by the HTSGQBO.

[2] A. Alessandri and F. Boem. State observers for systems subject to bounded disturbances using quadratic boundedness. IEEE Trans. on Automatic Control, 65(12):5352-5359, 2020.

[3] A. Alessandri, F. Boem, and T. Parisini. Model-based fault detection and estimation for linear time invariant and piecewise affine systems by using quadratic boundedness. In 57th IEEE Conference on Decision and Control, pages 55625567, Miami, USA, 2018.

[4] A. Alessandri and L. Zaccarian. Stubborn state observers for linear time-invariant systems. Automatica, 88:1-9, 2018.

[5] V. Andrieu, C. Prieur, S. Tarbouriech, and L. Zaccarian. A hybrid scheme for reducing peaking in high-gain observers for a class of nonlinear systems. Automatica, 72:138 - 146, 2016.

[6] D. Astolfi, P. Bernard, R. Postoyan, and L. Marconi. Constrained state estimation for nonlinear systems: a redesign approach based on convexity. IEEE Trans. on Automatic Control, to appear, 2021.

[7] D. Astolfi, R. Postoyan, and D. Nešić. Uniting observers. IEEE Trans. on Automatic Control, 65(7):2867-2882, 2020.

[8] F.J. Bejarano, L. Fridman, and A. Poznyak. Unknown input and state estimation for unobservable systems. SIAM Journal on Control and Optimization, 48(2):1155-1178, 2009.

[9] P. Bernard and L. Marconi. Hybrid implementation of observers in plant's coordinates with a finite number of approximate inversions and global convergence. Automatica, 111:108654, 2020.

[10] P. Bernard and R.G. Sanfelice. Observers for hybrid systems with linear flow/jump maps and known jump times. In 57th IEEE Conference on Decision and Control, pages 3140-3145, Miami, USA, 2018.

[11] F. Blanchini. Set invariance in control. Automatica, 35(11):1747 - 1767, 1999.

[12] F. Blanchini and S. Miani. Set-Theoretic Methods in Control. Birkhäuser Basel, 1st edition, 2007.

[13] C. Bogani, M.G. Gasparo, and A. Papini. Generalized pattern search methods for a class of nonsmooth optimization problems with structure. J. Comput. Applied Math., 229(1):283-293, 2009.

[14] S. Boyd, L. El Ghaoui, E. Feron, and V. Balakrishnan. Linear Matrix Inequalities in System and Control Theory, volume 15 of Studies in Applied Mathematics. SIAM, Philadelphia, PA, 1994.

[15] M. Brockman and M. Corless. Quadratic boundedness of nominally linear systems. Int. Journal of Control, 71(6):1105-1117, 1998.

[16] J. Chai and R.G. Sanfelice. Forward invariance of sets for hybrid dynamical systems (Part I). IEEE Trans. on Automatic Control, 64(6):2426-2441, 2019.

[17] D. Efimov, A. Polyakov, A. Levant, and W. Perruquetti. Convergence acceleration for observers by gain commutation. Int. Journal of Control, 91(9):2009-2018, 2018.

[18] F. Ferrante, F. Gouaisbaut, R.G. Sanfelice, and S. Tarbouriech. State estimation of linear systems in the presence of sporadic measurements. Automatica, 73:101-109, 2016.

[19] L. Fridman, J. Davila, and A. Levant. High-order slidingmode observation for linear systems with unknown inputs. Nonlinear Analysis: Hybrid Systems, 5(2):189-205, 2011.

[20] R. Goebel, R.G. Sanfelice, and A.R. Teel. Hybrid Dynamical Systems: Modeling, Stability, and Robustness. Princeton University Press, 2012.

[21] M. Johansson and A. Rantzer. Computation of piecewise quadratic Lyapunov functions for hybrid systems. IEEE Trans. on Automatic Control, 43(4):555-559, 1998.

[22] Y. Li and R.G. Sanfelice. A finite-time convergent observer with robustness to piecewise-constant measurement noise. Automatica, 57:222 - 230, 2015.

[23] Y. Liu. Switching observer design for uncertain nonlinear systems. IEEE Trans. on Automatic Control, 42(12):16991703, 1997.

[24] J. Löfberg. Yalmip: A toolbox for modeling and optimization in MATLAB. In Proceedings of the CACSD Conf., pages 284-289, Taipei, Taiwan, 2004.

[25] D.Q. Mayne, R.W. Grainger, and G.C. Goodwin. Nonlinear filters for linear signal models. IEE Proceedings - Control Theory and Applications, 144:281-286, 1997.

[26] D. Paesa, A. Baños, and C. Sagues. Optimal reset adaptive observer design. Systems \& Control Letters, 60(10):877-883, 2011.

[27] R. Rajamani, W. Jeon, H. Movahedi, and A. Zemouche. On the need for switched-gain observers for non-monotonic nonlinear systems. Automatica, 114:108814, 2020.

[28] H. Ríos and A.R. Teel. A hybrid fixed-time observer for state estimation of linear systems. Automatica, 87:103 - 112, 2018.

[29] H. Ríos, J. Davila, and A.R. Teel. Linear hybrid systems with periodic jumps: A notion of strong observability and strong detectability. IEEE Trans. on Automatic Control, 65(6):2640-2646, 2020.

[30] H. Ríos, J. Dávila, and A.R. Teel. State estimation for linear hybrid systems with periodic jumps and unknown inputs. Int. Journal of Robust and Nonlinear Control, 30(15):59665988,2020

[31] R. Sanfelice, D. Copp, and P. Nanez. A toolbox for simulation of hybrid systems in Matlab/Simulink: Hybrid equations (HyEQ) toolbox. In Proc. of the 16th International Conference on Hybrid Systems: Computation and Control, HSCC'13, page 101-106, New York, NY, USA, 2013. Association for Computing Machinery.

[32] R.G. Sanfelice. Hybrid Feedback Control. Princeton University Press, New Jersey, 2021. 